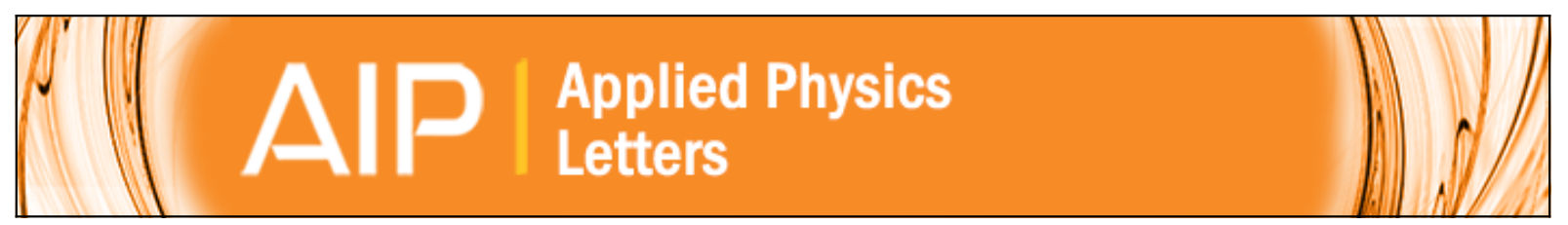

\title{
Temperature-dependent elastic properties of Ti1-xAlxN alloys
}

Nina Shulumba, Olle Hellman, Lina Rogström, Zamaan Raza, Ferenc Tasnádi, Igor A. Abrikosov, and Magnus Odén

Citation: Applied Physics Letters 107, 231901 (2015); doi: 10.1063/1.4936896

View online: http://dx.doi.org/10.1063/1.4936896

View Table of Contents: http://scitation.aip.org/content/aip/journal/apl/107/23?ver=pdfcov

Published by the AIP Publishing

\section{Articles you may be interested in}

Temperature-dependence of structural and mechanical properties of TiB2: A first principle investigation

J. Appl. Phys. 117, 225902 (2015); 10.1063/1.4922408

Ab-initio aprroach to the electronic, structural, elastic, and finite-temperature thermodynamic properties of Ti2AX $(\mathrm{A}=\mathrm{Al}$ or $\mathrm{Ga}$ and $\mathrm{X}=\mathrm{C}$ or $\mathrm{N})$

J. Appl. Phys. 110, 093504 (2011); 10.1063/1.3652768

Decomposition pathways in age hardening of Ti-Al-N films

J. Appl. Phys. 110, 023515 (2011); 10.1063/1.3610451

An ab initio study of the elastic behavior of single crystal group (IV) diborides at elevated temperatures Appl. Phys. Lett. 97, 211908 (2010); 10.1063/1.3514238

Ab initio calculated binodal and spinodal of cubic Ti $1-x \mathrm{Al} \times \mathrm{N}$

Appl. Phys. Lett. 88, 071922 (2006); 10.1063/1.2177630

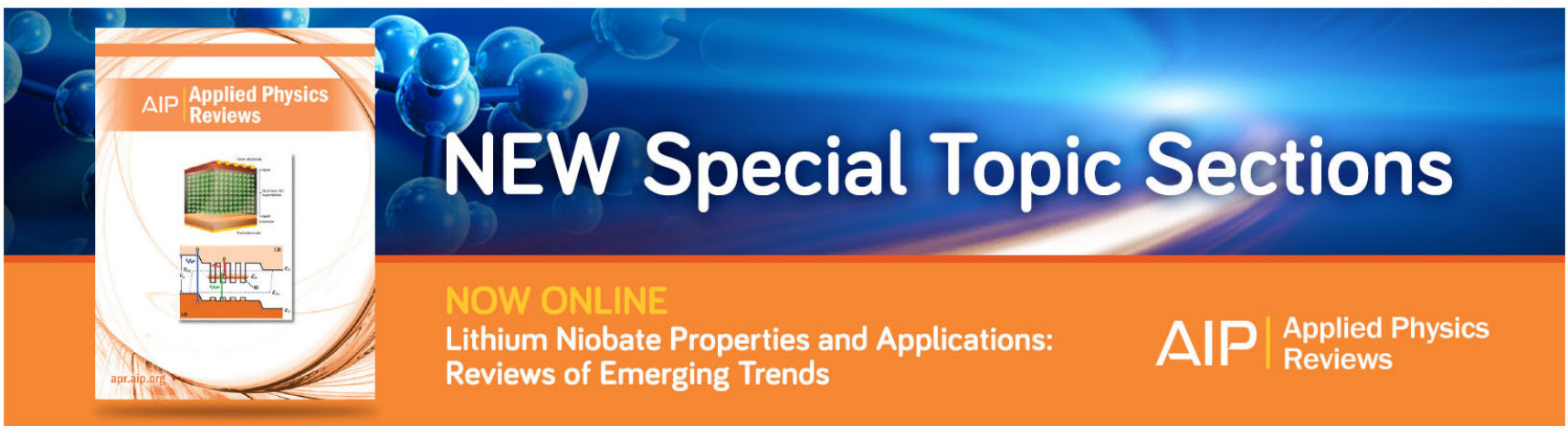




\title{
Temperature-dependent elastic properties of $\mathrm{Ti}_{1-x} \mathrm{Al}_{x} \mathrm{~N}$ alloys
}

\author{
Nina Shulumba, ${ }^{1,2}$ Olle Hellman, ${ }^{3,1}$ Lina Rogström, ${ }^{1}$ Zamaan Raza, ${ }^{1}$ Ferenc Tasnádi, ${ }^{1}$ \\ Igor A. Abrikosov, ${ }^{1,4,5}$ and Magnus Odén ${ }^{1}$ \\ ${ }_{1}^{1}$ Department of Physics, Chemistry, and Biology (IFM), Linköping University, SE-581 83 Linköping, Sweden \\ ${ }^{2}$ Functional Materials, Saarland University, D-66123 Saarbrücken, Germany \\ ${ }^{3}$ Division of Engineering and Applied Science, California Institute of Technology, Pasadena, \\ California 91125, USA \\ ${ }^{4}$ Materials Modeling and Development Laboratory, NUST “MISIS,” 119049 Moscow, Russia \\ ${ }^{5}$ LACOMAS Laboratory, Tomsk State University, 634050 Tomsk, Russia
}

(Received 26 September 2015; accepted 19 November 2015; published online 8 December 2015)

\begin{abstract}
$\mathrm{Ti}_{1-x} \mathrm{Al}_{x} \mathrm{~N}$ is a technologically important alloy that undergoes a process of high temperature agehardening that is strongly influenced by its elastic properties. We have performed first principles calculations of the elastic constants and anisotropy using the symmetry imposed force constant temperature dependent effective potential method, which include lattice vibrations and therefore the effects of temperature, including thermal expansion and intrinsic anharmonicity. These are compared with in situ high temperature x-ray diffraction measurements of the lattice parameter. We show that anharmonic effects are crucial to the recovery of finite temperature elasticity. The effects of thermal expansion and intrinsic anharmonicity on the elastic constants are of the same order, and cannot be considered separately. Furthermore, the effect of thermal expansion on elastic constants is such that the volume change induced by zero point motion has a significant effect. For TiAlN, the elastic constants soften non-uniformly with temperature: $C_{11}$ decreases substantially when the temperature increases for all compositions, resulting in an increased anisotropy. These findings suggest that an increased $\mathrm{Al}$ content and annealing at higher temperatures will result in a harder alloy. (C) 2015 AIP Publishing LLC. [http://dx.doi.org/10.1063/1.4936896]
\end{abstract}

When optimizing the performance of alloys used in high temperature applications, it is crucial to understand how temperature affects their elastic properties. In the case of $\mathrm{Ti}_{1-x} \mathrm{Al}_{x} \mathrm{~N}$ alloys, a frequently used system for hard-coating applications, annealing at $1300 \mathrm{~K}$ causes age hardening ${ }^{1-3}$ due to an isostructural spinodal decomposition into coherent cubic Al- and Ti-enriched $\mathrm{Ti}_{1-x} \mathrm{Al}_{x} \mathrm{~N}$ domains. The microstructural evolution during decomposition is strongly affected by chemical composition and elastic anisotropy. ${ }^{4,5}$ Thermal expansion and stiffness differences cause strain in the microstructure, which can be locally magnified by the elastic anisotropy and affect the mechanical performance of such alloys. ${ }^{6,7}$ We seek to elucidate the atomistic origins of the high temperature elastic anisotropy.

The elastic constants can be conveniently calculated from first principles at zero temperature. In this way, a strong compositional $(x)$ dependence has been demonstrated ${ }^{8,9}$ with a change in excess of $50 \%^{5}$ as the $\mathrm{Al}$ content increases. However, static calculations neglect the effects of temperature and lattice vibrations on elasticity, and in an industrial context, the operational temperature of these coatings is around $1300 \mathrm{~K}$.

In this letter, we use $a b$ initio Born-Oppenheimer molecular dynamics (BOMD) in the framework of density functional theory (DFT) to predict the influence of temperature on the elastic properties of disordered $\mathrm{Ti}_{1-x} \mathrm{Al}_{x} \mathrm{~N}$. We propose a method that allows one to determine the elastic behavior, including anharmonic effects, of the disordered alloy $\mathrm{Ti}_{1-x} \mathrm{Al}_{x} \mathrm{~N}$ over a wide range of temperatures and compositions. We show that the elastic behaviour changes with temperature, and that this change is of the same order as the compositional dependence. The elastic properties for thin films alloys are difficult to measure; therefore, we use our method to predict the elastic properties of the notoriously awkward material $\mathrm{Ti}_{1-x} \mathrm{Al}_{x} \mathrm{~N}$.

We first note that in a perfectly harmonic crystal, the elastic constants are temperature-independent. ${ }^{10}$ The anharmonic contributions can roughly be partitioned into two contributions: thermal expansion and intrinsic anharmonicity. The former is easier to quantify experimentally and theoretically than the latter. ${ }^{11,12}$ Statically, but including thermal expansion, the elastic constants are ${ }^{13,14}$

$$
C_{i j, k l}^{\mathrm{QH}}(T) \approx C_{i j, k l}^{0}(V(T)) .
$$

Here, $C^{0}$ denotes the $0 \mathrm{~K}$ elastic constants, calculated at the volume $V$, which corresponds to temperature $T$.

To deal with the intrinsic anharmonicity, we note the correspondence between long-wavelength phonons and elastic constants: ${ }^{15,16}$ the elastic constants are a function of the derivative of the acoustic modes of the phonon dispersion at small wavevectors. As the temperature increases, the phonon frequencies will shift to lower values due to anharmonicity. ${ }^{17}$ If we observe a shift in phonon frequencies relative to the static calculations, there is an anharmonicity-induced temperature dependence in the elastic constants. In this case, we can use their volume/temperature dependence, expressed in terms of the interatomic force constants ${ }^{12}$

$$
C_{i j, k l}^{\mathrm{ph}}(V, T)=-\frac{1}{2 V(T)} \sum_{n} \Phi_{i j}^{0 n}(V, T) r_{k}^{n} r_{l}^{n} .
$$


Here, $\Phi_{i j}^{0 n}(V, T)$ is the explicitly temperature and volume dependent interatomic force constant between atoms $i$ and $j$ situated in unit cell $n$, and $r_{k}^{n}$ is the position of atom $k$ in unit cell $n$, all taken with respect to a reference unit cell 0 .

Using the temperature dependent effective potential (TDEP) method, ${ }^{18-20}$ one can calculate the finite temperature phonon spectra, which implicitly include renormalization to all orders of anharmonicity, and thus derive the force constants, which we use to obtain the elastic constants from Eq. (2). However, this is a real space sum and can be numerically ill-conditioned when it comes to absolute values due to finite size effects. We do, however, know that TDEP can accurately determine temperature dependence of phonon frequencies, ${ }^{21,22}$ so we use the finite temperature behaviour of the force constants to get a general expression

$$
C_{i j, k l}(V, T)=C_{i j, k l}^{\mathrm{stat}}(V, 0) \frac{C_{i j, k l}^{\mathrm{ph}}(V, T)}{C_{i j, k l}^{\mathrm{ph}}(V, 0)} .
$$

This enables us to calculate finite temperature elastic constants at a fraction of the computational cost of ab initio molecular dynamics (AIMD) simulations in which the cell is distorted; we can also use the same data to compute free energies at no additional expense. Moreover, within this scheme, the origin of the thermal behaviour of the elastic constants can be easily elucidated, whether it is from thermal expansion or intrinsic anharmonicity. The accuracy and reliability of the scheme is demonstrated below.

Finite temperature simulations were performed using ab initio molecular dynamics in the canonical (NVT) ensemble on a grid of compositions, temperatures, and volumes. Forces were computed at the DFT level using the projector-augmented wave (PAW) method $^{23}$ as implemented in VASP, ${ }^{24-27}$ with the electronic contribution to the entropy given by the Mermin functional. ${ }^{28}$ The temperature was controlled by a Nosé thermostat. ${ }^{29,30}$ The plane wave energy cutoff was set to $600 \mathrm{eV}$ and the electronic exchange-correlation energy computed using the PerdewBurke-Ernzerhof functional. ${ }^{31}$ The configurational entropy was estimated using the mean-field approximation. ${ }^{32}$ To simulate a random alloy with B1 symmetry, we used a special quasirandom structure (SQS) approach. ${ }^{33}$ A 128 atom SQS $(4 \times 4 \times 4)$ was constructed, in which 64 atoms were metal and 64 nitrogen. For $\mathrm{T}=0 \mathrm{~K}$, elastic constants, and total energies, the Brillouin zone (BZ) was sampled with a $6 \times 6 \times 6$ Monkhorst-Pack grid of k-points. ${ }^{34}$ For each volume, temperature and concentration BOMD was performed for about 10000 time steps of $1 \mathrm{fs}$ after equilibration, using only the $\Gamma$-point for BZ integration. A set of 200 uncorrelated snapshots was selected, and the energies were recomputed with a $3 \times 3 \times 3 \mathrm{k}$-point mesh to ensure convergence. From these simulations, we extracted the effective interatomic force constants by means of TDEP, which were used to calculate the phonon density of states and the vibrational contribution to Helmholtz free energy. Using $p=-d F / d V$, the volume-temperature-concentration space was transformed to a pressure-temperature-concentration space. The irreducible components of the force constants were interpolated along the $P=0$ isobar, and substituted into (3) to obtain the elastic constants as a function of temperature. The elastic constants $\bar{C}_{11}, \bar{C}_{12}$, and $\bar{C}_{44}$ for alloys were calculated as an average of 9 elastic tensor elements. ${ }^{35}$

The temperature dependence of the lattice parameters contributes substantially to the temperature dependence of the elastic constants and is easier to measure and compare with our theoretical results. We therefore experimentally determined the lattice parameters as a function of temperature for $\mathrm{Ti}_{1-x} \mathrm{Al}_{x} \mathrm{~N}$ alloys $\left(\mathrm{TiN}, \mathrm{Ti}_{75} \mathrm{Al}_{25} \mathrm{~N}, \mathrm{Ti}_{50} \mathrm{Al}_{50} \mathrm{~N}\right.$, and $\mathrm{Ti}_{33} \mathrm{Al}_{67} \mathrm{~N}$ ), by in situ high temperature $\mathrm{x}$-ray diffraction (HT-XRD) during annealing. $\mathrm{Ti}_{1-x} \mathrm{Al}_{x} \mathrm{~N}$ coatings were grown by arc evaporation and removed from their substrates to obtain powder samples. The experiments were performed at beamline P07B, Petra III using a $53.7 \mathrm{keV}$ x-ray beam. The details on sample preparation and experimental setup are available in Ref. 36. The samples were annealed under vacuum, and two-dimensional diffraction patterns were collected in transmission geometry. The powder samples were first annealed for $2 \mathrm{~h}$ at $873 \mathrm{~K}$ to annihilate point defects. Subsequently, the temperature was reduced in steps of $75 \mathrm{~K}$, and the sample was held for $15 \mathrm{~min}$ at each temperature before the diffraction pattern was recorded. The plane spacing was determined for each temperature and the corresponding thermal expansion coefficient was extracted for each crystallographic direction $(111,200,220,311$, and 222) using a linear fit to the data. For each sample, an average thermal expansion coefficient was calculated from the different crystallographic directions. The relative theoretical and experimental thermal expansions are compared in Fig. 1 for different compositions of the $\mathrm{Ti}_{1-x} \mathrm{Al}_{x} \mathrm{~N}$ alloy, showing a good agreement. The experimental and theoretical thermal expansions for $\mathrm{Ti}_{1-x} \mathrm{Al}_{x} \mathrm{~N}$ alloys behave linearly within the experimental range of temperatures in Fig. 1 and deviate at higher temperatures due to strong temperature-induced anharmonicity.

Fig. 1 demonstrates that the lattice parameter of $\mathrm{Ti}_{1-x} \mathrm{Al}_{x} \mathrm{~N}$ increases by $2 \%-2.5 \%$ at $2000 \mathrm{~K}$, depending on the $\mathrm{Al}$ content. Using Eq. (1) and the elastic constants calculated using the established method of distorting the cell at zero temperature, ${ }^{37}$ we see a strong volume dependence in $C_{11}$ and weaker dependences in $C_{12}$ and $C_{44}$ (Fig. 2). The volume dependence of $C_{11}$ is so strong that the (usually negligible) effect of zero point motion on the lattice parameter decreases $C_{11}$ by $6 \%$ at zero temperature, from 609 to $577 \mathrm{GPa}$, and this is from a mere $0.3 \%$ change in lattice parameter. We conclude that the quantum mechanical zeropoint motion cannot be ignored below the Debye temperature. We now turn to the effect of temperature on elastic constants. In the quasiharmonic approximation (including thermal expansion but not anharmonicity), the elastic constants have a significant concentration $(x)$ dependence. $C_{11}$ softens by $\sim 22 \%$ in TiN, compared with $\sim 27 \%$ in cubic AlN. In absolute terms, $C_{11}$ decreases by $\sim 127 \mathrm{GPa}$ in TiN compared with $\sim 87 \mathrm{GPa}$ in AlN.

Moving to a higher level of theory and considering intrinsic anharmonic effects as described by Eq. (3), we see that the change in elastic constants due to anharmonicity is similar in magnitude to the change due to thermal expansion, as illustrated in Fig. 2. These results are in line with previous studies for $\mathrm{TiN}^{38}{ }^{38}$ with a significant softening of all elastic 

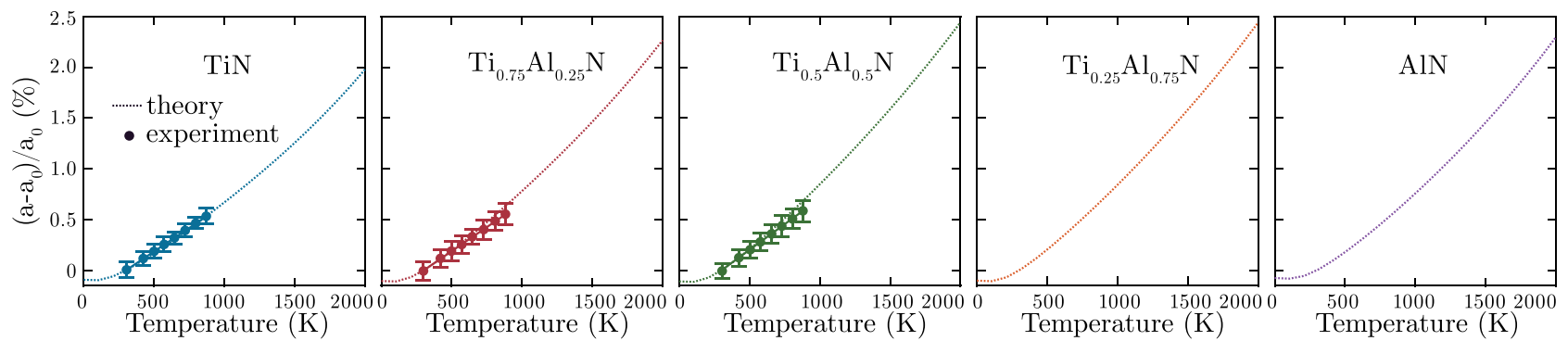

FIG. 1. Behaviour of the lattice parameter $a$ as expressed by the ratio $\left(a-a_{0}\right) / a_{0}$ as a function of temperature, for different compositions of a Ti $i_{1-x} \mathrm{Al}_{x} \mathrm{~N}$ alloy. The reference lattice parameter $\mathrm{a}_{0}$ is taken at $300 \mathrm{~K}$ for both experimental and theoretical cases. The experimental results were obtained with HTXRD.
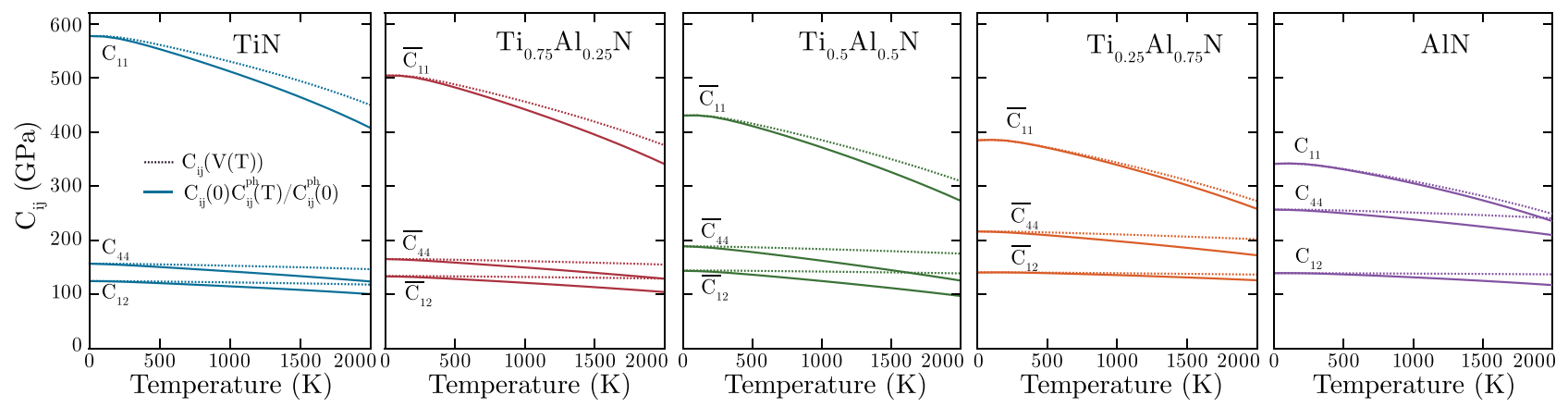

FIG. 2. Elastic constants as a function of temperature and composition for disordered $\mathrm{Ti}_{1-x} \mathrm{Al}_{x} \mathrm{~N}$. The dotted lines represent static elastic constants including thermal expansion, calculated using Eq. (1). The solid lines correspond to elastic constants calculated using our new method, as expressed in Eq. (3), including anharmonicity and thermal expansion. For the disordered alloy, $\bar{C}$ indicates symmetry averaged elastic constants.

constants. For TiN, our results agree well with the values obtained by full AIMD simulations by Steneteg et al. ${ }^{38} \mathrm{We}$ view this as a strong justification for the reliability and accuracy of the scheme based on the use of Eqs. (2) and (3). The divergence of the static calculations including thermal expansion demonstrates that first, such an approach is unreliable in general, since there are different trends for different compositions and elastic constants, and second that it is not possible to decouple the effects of thermal expansion and intrinsic anharmonicity. We note, however, that there is some disagreement between the two computational schemes below the Debye temperature ( $636 \mathrm{~K}$ for TiN). This can be explained with the fact that in Ref. 38, the simulations were based on classical equations of motions for atoms, whereas this work includes quantum effects on the vibrational free energy.

The temperature-induced softening of elastic constants is not uniform between tensorial components. This is quantified by the Zener anisotropy ratio $\mathrm{A}_{z}=2 \mathrm{C}_{44} /\left(\mathrm{C}_{11}-\mathrm{C}_{12}\right) .{ }^{39}$ Fig. 3 shows a contour plot of the Zener anisotropy as a function of temperature and concentration, and it demonstrates that $\mathrm{A}_{z}$ increases non-linearly with temperature and $\mathrm{Al}$ content. In the quasiharmonic approximation, the composition at which isotropy $\left(\mathrm{A}_{z}=1\right)$ occurs changes more rapidly with temperature compared with our method, which includes intrinsic anharmonicity. As a result, the quasiharmonic approximation underestimates the $\mathrm{Al}$ concentration at which isotropy occurs.

This anisotropy has a substantial impact on the microstructure evolution and the correlated age hardening of the thin films. Johansson Jöesaar et al. ${ }^{6}$ showed that low $\mathrm{Al}$ content in $\mathrm{Ti}_{1-x} \mathrm{Al}_{x} \mathrm{~N}$ coatings $\left(\mathrm{Ti}_{0.69} \mathrm{Al}_{0.31} \mathrm{~N}\right)$ demonstrates a low age hardening effect in comparison to the coatings with a high $\mathrm{Al}$ content $\left(\mathrm{Ti}_{0.34} \mathrm{Al}_{0.66} \mathrm{~N}\right)$. This is explained by the evolving microstructure during spinodal decomposition, where the higher anisotropy in the high $\mathrm{Al}$ content coatings results in more elongated Al- and Ti-enriched domains. This is believed to improve the hardness. ${ }^{7}$

In summary, we investigated thermal properties of $\mathrm{Ti}_{1-x} \mathrm{Al}_{x} \mathrm{~N}$ alloys by means of ab initio theoretical simulations and experiment. The calculated thermal expansion of $\mathrm{Ti}_{1-x} \mathrm{Al}_{x} \mathrm{~N}$ alloys is in good agreement with HT-XRD measurements. We calculated the elastic constants of a random

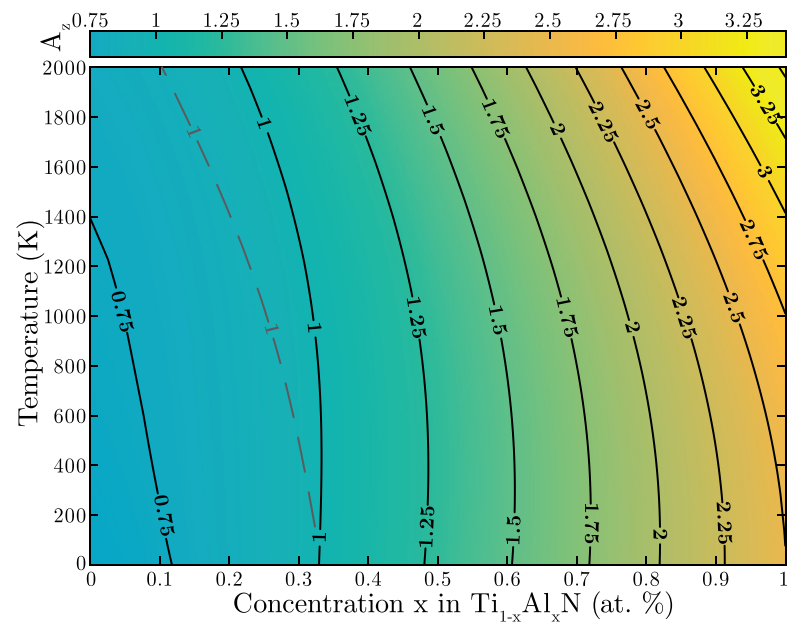

FIG. 3. Contour plot showing the Zener anisotropy as a function of temperature and $\mathrm{Al}$ content. The solid lines are contours of $\mathrm{A}_{z}$, calculated with anharmonic temperature dependent elastic constants. The static $\mathrm{A}_{z}$ including thermal expansion calculated using Equation (1) is shown with dashed lines, illustrating the underestimation in $\mathrm{A}_{z}$ if anharmonic effects are neglected. 
$\mathrm{Ti}_{1-x} \mathrm{Al}_{x} \mathrm{~N}$ alloy as a function of composition over a wide range of temperatures, demonstrating that the elastic anisotropy increases with temperature. We find that anharmonic effects and the thermal expansion are of equal importance when determining the elasticity at finite temperatures. We emphasize the importance of zero-point motion when elastic constants are determined, in spite of their relatively minor effect on the lattice constant.

The relationships between atomic structure, microstructure, and mechanical properties are complex and closely linked to lattice dynamics. Undercooled cubic $\mathrm{Ti}_{1_{x}} \mathrm{Al}_{x} \mathrm{~N}$ alloys having isotropic elastic constants decompose into a microstructure of domains that are notionally spherical, whereas alloys with high elastic anisotropy have elongated irregular shaped domains ${ }^{40}$ giving rise to an internal stress state and domain interface structure ${ }^{41}$ that are more effective in terms of obstructing dislocation motion, i.e., giving a higher hardness. Building on this notion, we identify two strategies for improving high temperature hardness of $\mathrm{Ti}_{1_{x}} \mathrm{Al}_{x} \mathrm{~N}$ alloys. The first is to use Al-rich alloys, which have a higher elastic anisotropy, as shown in Fig. 3. The second is to have the decomposition taking place at as high temperature as possible, which also increases the elastic anisotropy. The upper temperature limit for the second approach is given by the detrimental phase transformation of cubic to hexagonal AlN at approximately $1300 \mathrm{~K} .{ }^{36}$ However, successful attempts to push this transformation temperature up, e.g., by additional alloying elements ${ }^{42}$ and multilayer structures, ${ }^{43}$ suggest that substantial performance improvements of these alloys can be achieved by properly invoking high temperature elastic constants in the materials design.

Support from the Swedish Research Council (VR programs 621-2011-4426, 621-2012-4401, and 637-20137296), Swedish Foundation for Strategic Research (SSF programs RMA08-0069 (MultiFilms) and SRL10-0026), and VINNOVA (M-Era.net project 2013-02355( $\left.\mathrm{MC}^{2}\right)$ ) is acknowledged. N.S. acknowledges support from the Erasmus Mundus Joint European Doctoral Program DocMASE. I.A.A. acknowledges the support from the Grant of Ministry of Education and Science of the Russian Federation (Grant No. 14.Y26.31.0005) and Tomsk State University Academic D. I. Mendeleev Fund Program. All calculations were performed using the supercomputer resources of the Swedish National Infrastructure for Computing (SNIC), PDC and NSC centers. The use of Petra III was supported by a Rontgen-Angstrom Cluster frame grant (VR 2011-6505).

${ }^{1}$ A. Hörling, L. Hultman, M. Odén, J. Sjölén, and L. Karlsson, J. Vac. Sci. Technol., A 20, 1815 (2002).

${ }^{2}$ A. Knutsson, M. P. Johansson, P. O. Å. Persson, L. Hultman, and M. Odén, Appl. Phys. Lett. 93, 143110 (2008).

${ }^{3}$ P. H. Mayrhofer, A. Hörling, L. Karlsson, J. Sjölén, T. Larsson, C. Mitterer, and L. Hultman, Appl. Phys. Lett. 83, 2049 (2003).
${ }^{4}$ D. J. Seol, S. Y. Hu, Y. L. Li, J. Shen, K. H. Oh, and L. Q. Chen, Acta Mater. 51, 5173 (2003).

${ }^{5}$ F. Tasnádi, I. A. Abrikosov, L. Rogström, J. Almer, M. P. Johansson, and M. Odén, Appl. Phys. Lett. 97, 231902 (2010).

${ }^{6} \mathrm{M}$. P. Johansson Jöesaar, N. Norrby, J. Ullbrand, R. M'Saoubi, and M. Odén, Surf. Coat. Technol. 235, 181 (2013).

${ }^{7}$ B. Sonderegger and E. Kozeschnik, Scr. Mater. 66, 52 (2012).

${ }^{8}$ P. H. Mayrhofer, D. Music, and J. M. Schneider, J. Appl. Phys. 100, 094906 (2006).

${ }^{9}$ J. Von Pezold, A. Dick, M. Friák, and J. Neugebauer, Phys. Rev. B 81, 094203 (2010).

${ }^{10}$ A. G. McLellan, J. Phys. C 18, 5511 (1985).

${ }^{11}$ R. A. Cowley, Rep. Prog. Phys. 31, 123 (1968).

${ }^{12}$ G. Leibfried and W. Ludwig, Solid State Phys. (Academic Press, New York, 1961), Vol. 275.

${ }^{13}$ S.-L. Shang, H. Zhang, Y. Wang, and Z.-K. Liu, J. Phys.: Condens. Matter 22, 375403 (2010).

${ }^{14}$ Y. Wang, J. J. Wang, H. Zhang, V. R. Manga, S. L. Shang, L.-Q. Chen, and Z.-K. Liu, J. Phys.: Condens. Matter 22, 225404 (2010).

${ }^{15}$ B. Fultz, Prog. Mater. Sci. 55, 247 (2010).

${ }^{16}$ G. P. Srivastava, The Physics of Phonons (Taylor \& Francis, 1990).

${ }^{17}$ A. A. Maradudin and A. E. Fein, Phys. Rev. 128, 2589 (1962).

${ }^{18}$ O. Hellman, I. A. Abrikosov, and S. I. Simak, Phys. Rev. B 84, 180301 (2011).

${ }^{19}$ O. Hellman, P. Steneteg, I. A. Abrikosov, and S. I. Simak, Phys. Rev. B 87, 104111 (2013).

${ }^{20}$ N. Shulumba, Ph.D. thesis "Vibrations in Solids: From first principles lattice dynamics to high temperature phase stability," Linköping University, 2015 , p. 59.

${ }^{21}$ C. W. Li, O. Hellman, J. Ma, A. F. May, H. B. Cao, X. Chen, A. D. Christianson, G. Ehlers, D. J. Singh, B. C. Sales, and O. Delaire, Phys. Rev. Lett. 112, 175501 (2014).

${ }^{22}$ A. H. Romero, E. K. U. Gross, M. J. Verstraete, and O. Hellman, Phys. Rev. B 91, 214310 (2015).

${ }^{23}$ P. E. Blöchl, Phys. Rev. B 50, 17953 (1994).

${ }^{24}$ G. Kresse and J. Hafner, Phys. Rev. B 48, 13115 (1993).

${ }^{25}$ G. Kresse and J. Furthmüller, Phys. Rev. B 54, 11169 (1996).

${ }^{26}$ G. Kresse and J. Furthmüller, Comput. Mater. Sci. 6, 15 (1996).

${ }^{27}$ G. Kresse and D. Joubert, Phys. Rev. B 59, 1758 (1999).

${ }^{28}$ N. Mermin, Phys. Rev. 137, A1441 (1965).

${ }^{29}$ S. Nosé, Mol. Phys. 52, 255 (1984).

${ }^{30}$ W. Hoover, Phys. Rev. A 31, 1695 (1985).

${ }^{31}$ J. P. Perdew, K. Burke, and M. Ernzerhof, Phys. Rev. Lett. 77, 3865 (1996).

${ }^{32} \mathrm{G}$. Inden, Report on the Project Meeting CALPHAD V (Max-Planck Inst., Eisenforsch, Dusseldorf, 1976), p. 111.

${ }^{33}$ A. Zunger, S. H. Wei, L. G. Ferreira, and J. E. Bernard, Phys. Rev. Lett. 65, 353 (1990).

${ }^{34}$ J. D. Pack and H. J. Monkhorst, Phys. Rev. B 16, 1748 (1977).

${ }^{35}$ F. Tasnádi, M. Odén, and I. A. Abrikosov, Phys. Rev. B 85, 144112 (2012).

${ }^{36}$ N. Norrby, L. Rogström, M. Johansson-Jõesaar, N. Schell, and M. Odén, Acta Mater. 73, 205 (2014).

${ }^{37}$ G. Grimvall, Thermophysical Properties of Materials (Elsevier Science, 1999).

${ }^{38}$ P. Steneteg, O. Hellman, O. Y. Vekilova, N. Shulumba, F. Tasnádi, and I. A. Abrikosov, Phys. Rev. B 87, 094114 (2013).

${ }^{39} \mathrm{C}$. Zener, Elasticity and Anelasticity of Metals (University of Chicago Press, 1948).

${ }^{40}$ A. Knutsson, J. Ullbrand, L. Rogström, N. Norrby, L. J. S. Johnson, L. Hultman, J. Almer, M. P. Johansson Jöesaar, B. Jansson, and M. Odén, J. Appl. Phys. 113, 213518 (2013).

${ }^{41}$ K. Yalamanchili, I. C. Schramm, E. Jiménez-Piqué, L. Rogström, F. Mücklich, M. Odén, and N. Ghafoor, Acta Mater. 89, 22 (2015).

${ }^{42}$ H. Lind, R. Forsén, B. Alling, N. Ghafoor, F. Tasndi, M. P. Johansson, I. A. Abrikosov, and M. Odén, Appl. Phys. Lett. 99, 091903 (2011).

${ }^{43}$ A. Knutsson, M. P. Johansson, L. Karlsson, and M. Odén, J. Appl. Phys. 108, 44312 (2010). 